\title{
Conclusiones de las Ponencias y Debates del Coloquio Internacional sobre Ahorro de Energía celebrado en Budapest, organizado por la Sección Húngara de la Europrefab
}

Fernando Aguirre de Iraola, Doctor Arquitecto

Durante los días 10 y 11 de abril, el doctor Arquitecto F. Aguirre, director del ITEcc, estvuo en Budapest para presidir el Coloquio Internacional sobre "Ahorro de energía en la edificación», que había sido organizado por la sociedad europea para la promoción de la industrialización de la construcción, (EUROPREFAB), de la cual el profesor Aguirre es presidente.

Las reuniones tuvieron lugar en la Sociedad Húngara de Arquitectura y Urbanismo y su coordinación estuvo a cargo del Instituto Científico de la Construcción. Actuó como vicepresidente el vicepresidente de la EUROPREFAB y delegado de la sección húngara de ésta, doctor Pozsgai.

El tema general del coloquio ha sido de enorme interés, pues trata de resolver el acuciante problema mundial de la crisis energética, referido al sector de la edificación, en especial.

Las ponencias presentadas estuvieron a cargo de eminentes especialistas europeos, expertos en física aplicada a la construcción, procesos industrializados, energía solar y racionalización y economía de la construcción. Los nombres de los ponentes y títulos de las ponencias han sido los siguientes:

- Doctores Egyedi (András) y Szirányi, de Budapest: «Energy-saving in industrial building desing».

- Ingeniero Oravecz y Arquitecto Mrs. Fekecs, de Budapest: «How energy could be saved by design».

- Arquitecto Kohler, de Lausanne: «Energy budgets for building systems».

- Doctor Jech, de Praga: "Energy requirements of selected prefabricated building system and possibilities of energy economy in Czechoslovaquia».

- Doctores Jech, Sterba y Alexander D., de Praga: «Primary energy requirements in the production of precast reinforced concrete building components».

- Doctores Rehanek, Janous y Sála, de Praga: «Energy conservation Projekt for the heating of building in Czechoslovaquia».

- Ingenieros Bondil y Vandenkerckhove, de París: «Administrative and practical measures for building energy saving in France».

- Profesor Martorana, de Milán: «Regulations on energy saving in Italy».

- Profesor Byberg, de Copenhague: «Experiencies from low-energy housing desing with special regard to the heat storage capacity of concreter).

En resumen es de destacar la importancia del coloquio, pues las ideas contenidas en las ponencias y las resultantes de los interesantes debates sugeridos a continuación de la exposición de cada una de ellas revolucionan, en cierto aspecto, los conceptos acerca de los sistemas industrializados $y$, en general, del futuro de la construcción.

La exposición de las ponencias presentadas al coloquio internacional sobre "Ahorro de energía en la edificación» y los debates producidos al final de cada una de ellas entre los presentes y los demás participantes permiten llegar a una serie de consideraciones, alguna de las cuales no me atrevería a calificar de conclusiones, dado el carácter de novedad que tienen muchas de las ideas expuestas y la heterogeneidad de las intervenciones.

Sin embargo, se han podido comprobar el interés y la gran actualidad de los temas debatidos, así como la conciencia de que, en muchos aspectos, las nuevas ópticas de concebir el diseño y el proceso constructivo de los sistemas influirán notablemente en las construcciones industrializadas. 
Como principio general, puede afirmarse que la mayoría de los países miembros están fuertemente afectados por la crisis energética internacional, provocada por el constante aumento del precio del petróleo, y que, por lo tanto, hay que aprovechar al máximo las fuentes de energía, que en algunos países son francamente escasas.

En el orden del diseño, y demostrado que las medidas que se adopten para economizar energía provocarán sin duda métodos más económicos de construcción, las ponencias presentadas por la sección húngara son interesantes. La primera, del doctor Egyedi y del ingeniero Szyrányi, y que lleva el título "Energy-saving in industrial building desing», constituye un estudio comparativo sumamente útil. Aunque el planning del ahorro de energía de los edificios por medio del computador no está resuelto todavía, las alternativas analizadas tomando como base dos naves industriales similares llevan a la conclusión de que la comparación entre las construcciones portantes metálicas y de hormigón armado, y la comparación entre las fábricas de hormigón armado aislado y los de paneles sandwiches ligeros, sobre la base de costos y energía muestran que las construcciones de hormigón armado son los más económicos. El edificio metálico es ligeramente más costoso, pero la cantidad de energía invertida es mucho mayor.

La segunda ponencia húngara, titulada «How energy could be saved by desing», original del ingeniero Oravecz y de la arquitecto Mrs. Fekecs, tuvo un gran interés, especialmente bajo el punto de vista del diseño arquitectónico, pues el estudio de los parámetros volumétricos y superficiales conducen a un método gráfico que permite al proyectista seguir la influencia de las variables, con lo que es posible evitar los valores demasiado elevados de pérdidas por transmisión del calor. Constituye un serio trabajo de investigación, que demuestra la influencia de la distancia relativa y la altura de los edificios, de su entorno ecológico, de los vientos que inciden sobre sus superficies, etcétera (figs. 1 y 2).

La ponencia suiza, presentada por el arquitecto Kohler, expuso una filosofía original acerca de la estructura interna de un edificio, como principal característica de éste, y de la descomposición de un sistema constructivo en subsistemas. Dicha ponencia fue muy apreciada por los asistentes, y de ella nos atraveríamos a obtener las siguientes conclusiones:

1. El estudio actual del desarrollo de los sistemas constructivos se basa en la posibilidad del aislamiento de subsistemas, optimizando éstos y con ello al sistema completo.

2. La necesidad de reducir el consumo de energía implica un estudio global del edificio.

3. El presupuesto energético, como estudio global que incluye los costos de energía de los materiales y componentes, así como el plazo de ejecución del edificio, es un medio eficaz de controlar el costo de energía en cada nivel de la planificación del proceso constructivo.

4. Sobre la base de los presupuestos energéticos y del conocimiento de las principales interrelaciones de energía entre subsistemas, es posible definir un nuevo esquema de referencia para todos los subsistemas y desarrollar algunos de ellos.

\section{RELACION ENTRE EL INCREMENTO DE LA CONFIGURACION VOLUMETRICA Y LA SUPERFICIE ESPECIFICA DE UN EDIFICIO}

\section{$V$ (cubicación) A}

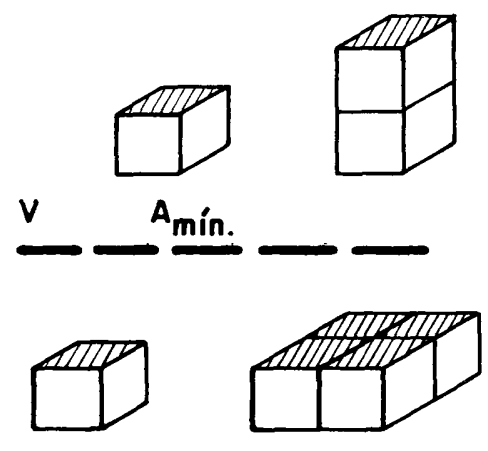

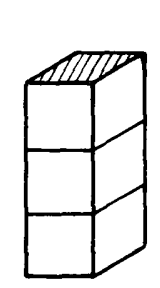
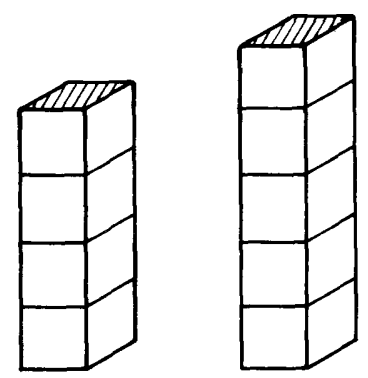
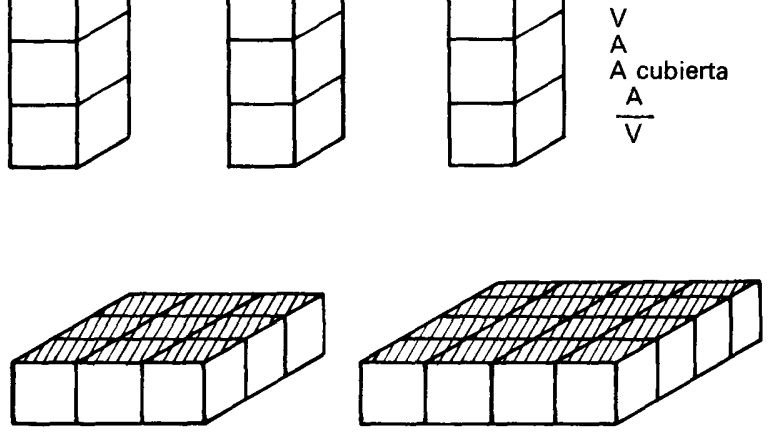

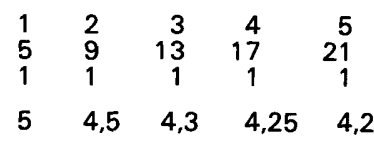

Fig. 1. 
5. Estamos sólo al comienzo de las medidas de ahorro de energía. Seguramente, en el futuro, el consumo de $150-170 \mathrm{~m}^{3} / \mathrm{m}^{2}$ año para la vivienda se verá reducida a la quinta parte.

Solamente las casas proyectadas y construidas teniendo muy en cuenta el problema energético serán capaces de satisfacer las futuras exigencias. La industrialización es el único medio de poder satisfacer estas exigencias a gran escala.

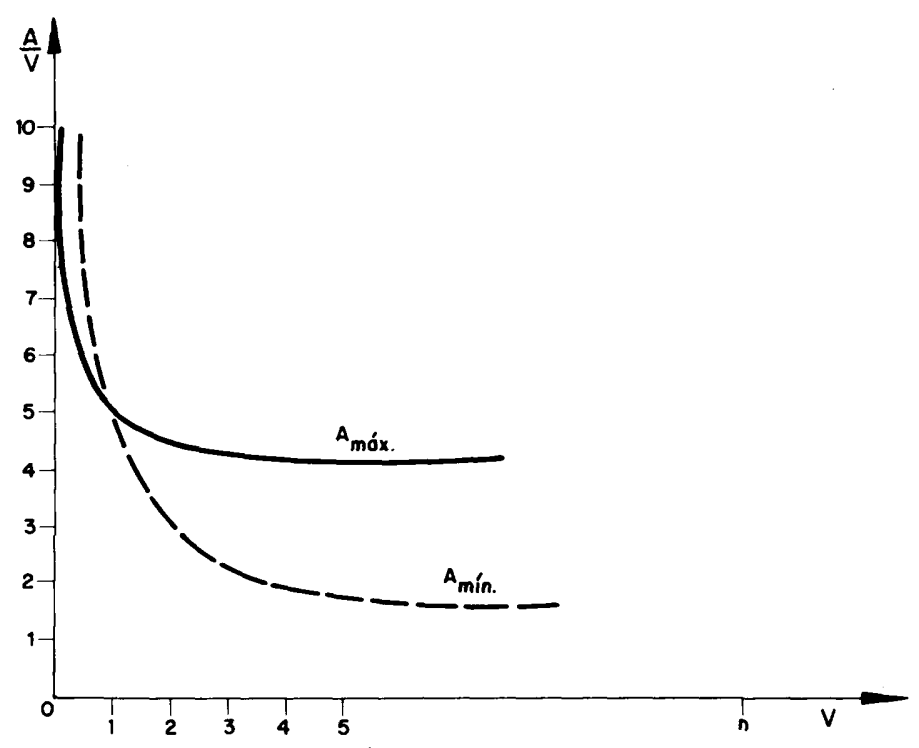

Fig. 2.

Al considerar el proceso total constructivo, en relación con el tema de la consenvación de la energía, es necesario el estudio de la energía primaria, que requiere la necesaria para la producción total, y que incluye la extracción y el proceso de elaboración de los materiales, a partir de las materias primas; la generación de la energía respectiva y su distribución, y la producción del propio elemento constructivo.

En este orden de ideas, la ponencia checa, original del doctor Jech, de Praga, que llevaba como título «Energy requirements of selected prefabricated building system and possibilities of energy economy in Czechoslovaquia», aportó datos muy interesantes y condujo a las siguientes conclusiones:

Para obtener economía mediante las exigencias energéticas en la construcción y la ejecución de edificios es preciso considerar las medidas siguientes:

- Reducción de las exigencias energéticas de los edificios mediante una optimización del proyecto estructural y una estricta conformidad con la tecnología de producción prescrita para sus componentes prefabricados.

- Aumento de las propiedades de aislamiento térmico de los edificios, tanto en su estructura como en sus elementos de cerramiento.

- Comprobación de la conformidad con la tecnología de producción prescrita para sus componentes prefabricados y con la construcción del edificio que integran, y control que asegure que la resistencia térmica de su estructura satisface las normas.

- Previsión de todas las fuentes de calor con dispositivos de control automático y de medida de consumo térmico, combinadas con medidas de suministro de calor y agua caliente, en las unidades de vivienda individuales.

Con referencia a la satisfacción uniforme de las experiencias energéticas primarias es aconsejable especificar de un modo más preciso el método de determinación de las exigencias energéticas de las materias primas $y$, en primer lugar, determinar las exigencias energéticas primarias normalizadas, para las unidades empleadas corrientemente en la práctica.

La segunda ponencia checa, de los doctores Alexander Doktor, Jech y Sterba, de Praga, después de un análisis, concluye que las exigencias energéticas primarias en la producción de los componentes de construcción prefabricados se hallan influenciados, en primer lugar, por: 


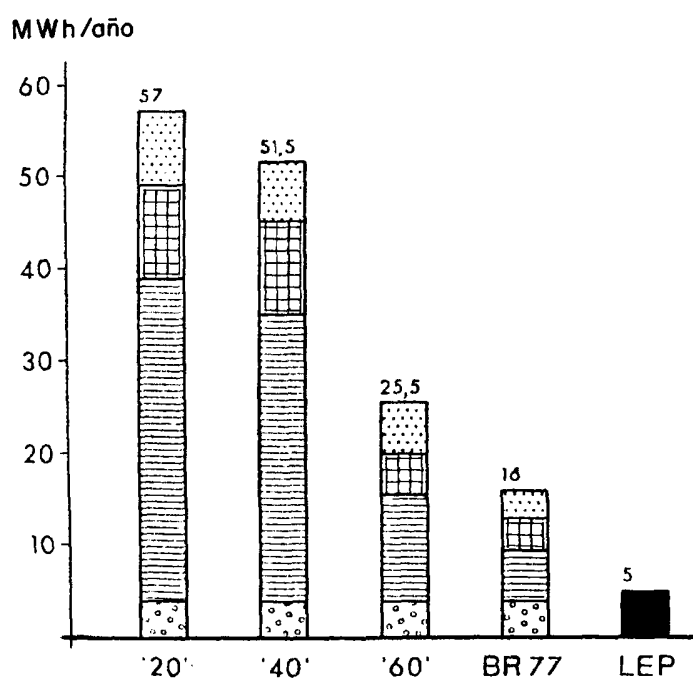

Ventilación

Pérdidas caloríficas a través de ventanas

Pérdidas coloríficas a través del resto de los cerramientos

Agua caliente
Consumo anual de energía para una típica vivienda unifamiliar danesa de $130 \mathrm{~m}^{2}$.

- Las exigencias energéticas indirectas incorporadas en el cemento y en el acero.

- Las exigencias energéticas directas del curado acelerado del hormigón, y que las medidas tendentes a lograr una economía energética debería dirigirse, en primer lugar, hacia aquellas disposiciones técnicas y tecnológicas que puedan incrementarse en plantas de prefabricados y que redunden en una máxima economía de energía primaria.

En relación a la energía consumida en el proceso edificatorio y en el tipo de utilización del edificio, la tercera ponencia presentada por Checoslovaquia (doctores Rehanek, Janous y Sála) bajo el título «Energy conservation Projekt for the heating of building in Czechoslovakia», preconiza las siguientes acciones, para asegurar el ahorro de energía:

- Preparación de proyectos ideales de estructuras interiores y exteriores.

- Previsión del tipo de proyecto de los sistemas estructurales en viviendas y edificios públicos.

La ponencia francesa, producida por los señores Bondil y Vandenkerckhove, de París, y que se titulaba "Administrative and practical measures for building energy saving in. France», aportó valiosa información sobre las normas y medidas adoptadas en este país, pudiéndose concluir de ello y de las discusiones que sugirió durante el coloquio que las medidas de ahorro de energía en Francia para la próxima década se basarán en el incremento de los métodos y materiales de aislamiento y control térmico, y que las normas serán muy rigurosas, aunque su aplicación requerirá tiempo y dinero, esencialmente en los edificios existentes.

En la línea de aportación francesa, el profesor S. Martorana, de Milán, en su trabajo «Regulations on energy saving in Itali», explica el desarrollo de algunos parámetros energéticos y sucio-económicos vigentes en Italia de 1963 a 1978, y puso de manifiesto las conveniencias del aumento del precio del petróleo en el sistema italiano. Demostró también que esta situación es similar en todos los países industrializados con economía de mercado.

De este análisis dedujo las medidas adoptadas en Italia y, en general, en otros países, subrayando, en el caso de Italia, que el suministro de energía para cada unidad de edificación depende de la zona climática $y$ de la relación entre la superficie y el volumen calentado. Además, comentó unas diapositivas que mostraron la investigación realizada por el Instituto de Física Técnica del Politécnico de Milán sobre el control del aislamiento mediante termografía infrarroja. Concluyó que la Industrialización de la construcción, mediante los sistemas cerrados, puede y debe contribuir a la conversión de los sistemas energéticos actuales en sistemas energéticos con bajo consumo de energía.

Por último, se expuso un excelente trabajo como resultado de una investigación sobre modelos a escala real: el profesor Byberg, de Copenhague, en su ponencia «Experiences from low-energy housing desing with special regard to the heat storage capacity of concreten, demostró que el empleo de 
construcciones pesadas con gran capacidad de almacenamiento térmico debe ser considerado como un esfuerzo para obtener buenas condiciones de confort antes que como materia de conservación de la energía. El procedimiento de cálculo y las diapositivas expuestas mostraron el interés y calidad de investigación.

En resumen: juzgamos que el interés despertado por el coloquio justificaría la celebración periódica de esta clase de reuniones, en el seno de la EUROPREFAB, pues cumplirán seguramente uno de los principales objetivos de esta Asociación.
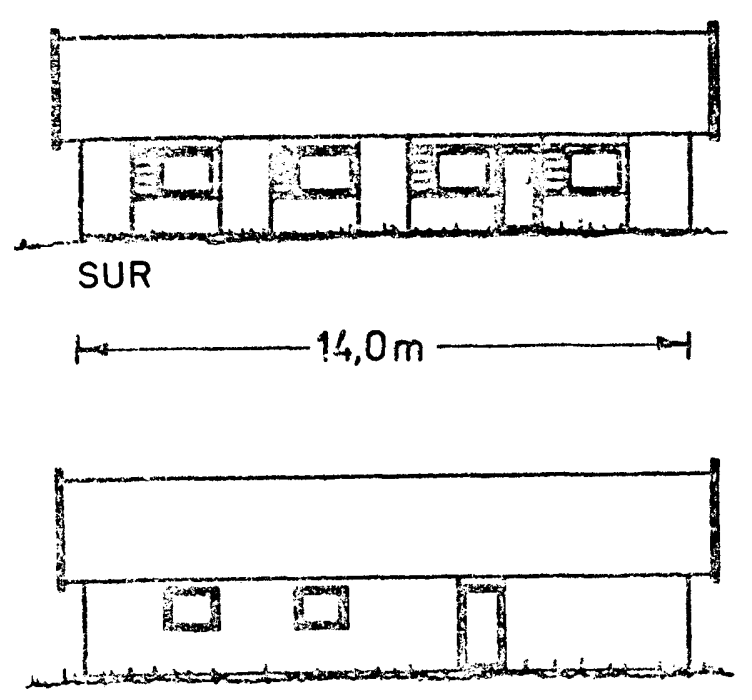

NORTE

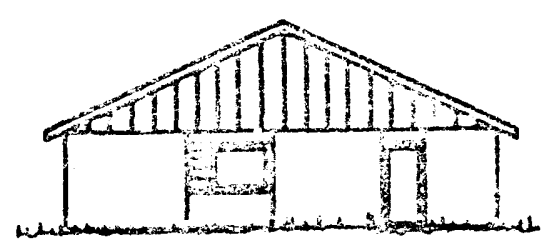

ESTE
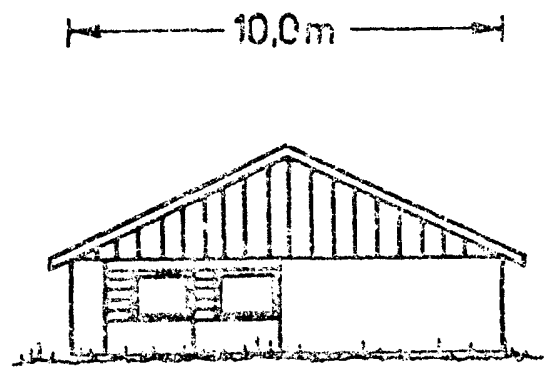

OESTE

Fig. 4.

Pérdidas de transmisión y ventilación para una típica casa unifamiliar danesa, de acuerdo con las normas de edificación de 1977

\begin{tabular}{|c|c|c|c|c|c|}
\hline Componentes & $\underset{m^{2}}{A}$ & $\underset{W / m^{2}{ }^{\circ} \mathrm{C}}{K}$ & $\begin{array}{l}K \cdot A \\
W /{ }^{\circ} \mathrm{C}\end{array}$ & $\underset{{ }^{\circ} \mathrm{C}}{\Delta t}$ & $\begin{array}{c}\text { Pérdidas caloríficas } \\
\qquad \mathbf{W}\end{array}$ \\
\hline \multicolumn{6}{|l|}{ Suelo } \\
\hline Parte interior & 84,4 & 0,3 & - & 12 & 304 \\
\hline Parte perimetral & 41,6 & 0,3 & 12,5 & 32 & 399 \\
\hline Tejado & 144,9 & 0,2 & 29,0 & 32 & 927 \\
\hline Ventanas $y$ puertas & 21,0 & 2,9 & 60,9 & 32 & 1.949 \\
\hline Ventilación & 4,2 & 2,0 & 8,4 & 32 & 269 \\
\hline Antepecho & 16,8 & 0,3 & 5,0 & 32 & 161 \\
\hline \multirow[t]{2}{*}{ Muro exterior } & 67,4 & 0,4 & 27,0 & 32 & 863 \\
\hline & & $\mathrm{W} /{ }^{\circ} \mathrm{C}$ & & W & 4.872 \\
\hline \multicolumn{4}{|l|}{ Transmisión al terreno } & 304 & W \\
\hline \multicolumn{4}{|c|}{ Transmisión al aire exterior } & 143,6 & $\mathrm{~W} /{ }^{\circ} \mathrm{C}$ \\
\hline \multicolumn{4}{|c|}{$\begin{array}{l}\text { Renovación de aire } 0,55 \mathrm{~h}^{-1}, \min .140 \mathrm{~m}^{3} / \mathrm{h} \\
\text { Ventilación: } \mathrm{m}^{3} / \mathrm{h} \times 0,34 \mathrm{Wh} / \mathrm{m}^{30} \mathrm{C}\end{array}$} & 51,9 & $\mathrm{~W} /{ }^{\circ} \mathrm{C}$ \\
\hline \multicolumn{2}{|l|}{ Superficie acristalada: } & $\begin{array}{l}\text { orte } \\
2 \mathrm{~m}^{2}\end{array}$ & 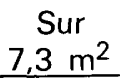 & $\begin{array}{c}\text { Este } \\
2,7 \mathrm{~m}^{2}\end{array}$ & $\begin{array}{l}\text { Oeste } \\
3 \mathrm{~m}^{2} \\
\end{array}$ \\
\hline
\end{tabular}



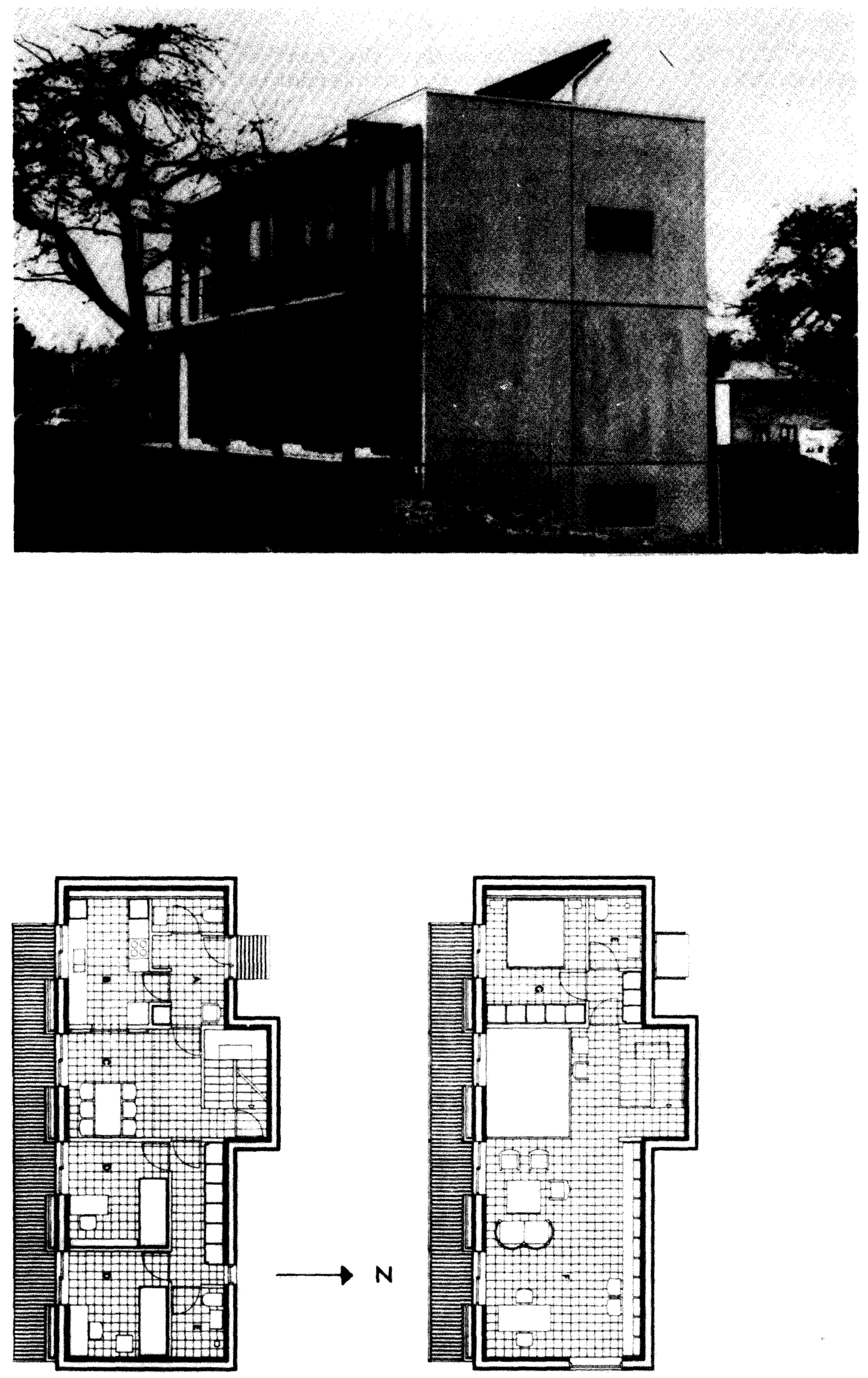

Fig. 5.

\section{planta baja}

planta primera 

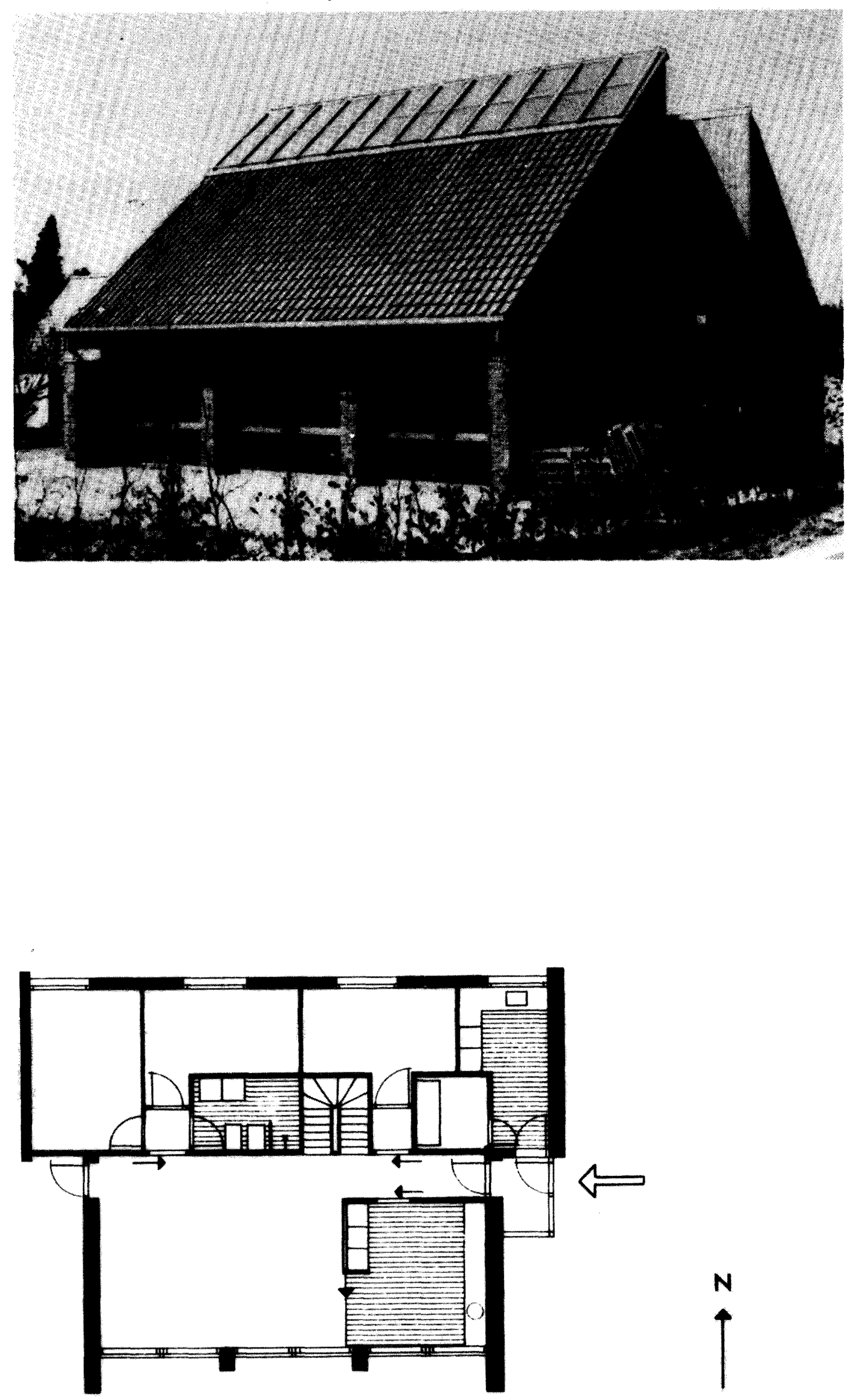

planta

Fig. 6. 